\title{
A CLASS OF ENTIRE FUNCTIONS WITH BOWL-LIKE SURFACES
}

\section{MINAKETAN DAS ${ }^{1}$}

1. Littlewood and Offord [1] have shown that for "almost all" entire functions of finite order $\rho>0$, the surface $u=\log ^{+}|f(x+i y)|$ in the three-dimensional $x, y, u$-space is like a bowl, i.e. outside small pits near the zeros, $u$ is of order of magnitude of $\log M(r, f)$ where

$$
M(r, f)=\operatorname{Sup}_{|z|=r}|f(z)| .
$$

However, very few of the special functions of analysis are of this type. In this note we show that a large class of functions of the form

$$
f(z)=\prod_{k=1}^{\infty}\left(1-\left(z / r_{k}\right)^{m k}\right)
$$

exhibit this behavior. The choice of the positive integers $m_{k}$ and the positive numbers $r_{k}$ can be varied in very wide limits. We shall only assume that

$$
\begin{aligned}
m_{k+1} & \geqq m_{k}, \\
\frac{r_{k+1}}{r_{k}}>1+\frac{\delta}{m_{k}} \quad \text { for some } \delta>0, & \\
n & =o\left(N\left(r_{n}\right)\right) \quad \text { as } n \rightarrow \infty,
\end{aligned}
$$

where

$$
N(r)=\sum_{r_{k} \leq r} m_{k} \log \left(r / r_{k}\right) .
$$

It is not hard to see that these conditions can be satisfied in such a way that $N(r)$ is of the order of magnitude $r^{p}$ for any assigned positive number $\rho$.

2. We show now that for $\left(r_{n-1} r_{n}\right)^{1 / 2} \leqq|z|=r \leqq r_{n}$,

(6) $\log |f(z)|=(1+o(1)) N(r)+\log \left|1-(z / r)^{m_{n}}\right| \quad(r \rightarrow \infty)$.

Now, for

$$
\left(r_{n-1} r_{n}\right)^{1 / 2} \leqq|z|=r<r_{n}
$$

Received by the editors April 22, 1964 and, in revised form, February 16, 1965.

${ }^{1}$ I wish to express my gratitude to the referee for his helpful suggestions and valuable criticism. 
we have

$$
\begin{aligned}
\left|\frac{r_{k}}{z}\right|^{m_{k}} & <\left(r_{k} / r_{k+1}\right)^{m_{k}} \quad(k<n-1) \\
& <\left(1+\frac{\delta}{m_{k}}\right)^{-m_{k}}<(1+\delta)^{-1}
\end{aligned}
$$

using (3). Similarly

$$
\left|r_{n-1} / z\right|^{m_{n-1}} \leqq\left(r_{n-1} / r_{n}\right)^{m_{n-1} / 2}<(1+\delta)^{-1 / 2} .
$$

Also, for $k>n$, by (2) and (3)

$$
\begin{aligned}
& \left|z / r_{k}\right|^{m_{k}}<\left(r_{n} / r_{n+1}\right)^{m_{n}} \cdot\left(r_{n+1} / r_{n+2}\right)^{m_{n+1}} \cdots\left(r_{k-1} / r_{k}\right)^{m_{k-1}} \\
& <(1+\delta)^{n-k} \text {. }
\end{aligned}
$$

By (7) and (8)

$$
\sum_{k=1}^{n-1} \log \left|1-\left(r_{k} / z\right)^{m_{k}}\right|=O(n)
$$

by (9),

$$
\sum_{k=n+1}^{\infty} \log \left|1-\left(z / r_{k}\right)^{m_{k}}\right|=O(1)
$$

Since

$$
\begin{aligned}
\log |f(z)|= & N(r)+\sum_{k=1}^{n-1} \log \left|1-\left(r_{k} / z\right)^{m_{k}}\right|+\log \left|1-\left(z / r_{n}\right)^{m_{n}}\right| \\
& +\sum_{k=n+1}^{\infty} \log \left|1-\left(z / r_{k}\right)^{m_{k}}\right|
\end{aligned}
$$

(6) follows from (10), (11) and (4).

For $r_{n} \leqq|z|=r \leqq\left(r_{n} r_{n+1}\right)^{1 / 2}$, one has

(12) $\log |f(z)|=(1+o(1)) N(r)+\log \left|1-\left(z / r_{n}\right)^{m_{n}}\right| \quad(r \rightarrow \infty)$.

It follows from (6) and (12) that

$$
\log |f(z)| \sim N(r)
$$

as $z \rightarrow \infty$ in any manner outside small circles round the zeros of $1-\left(z / r_{n}\right)^{m_{n}}$. It is enough to choose the radii of these circles equal to

$$
\left(r_{n} / m_{n}\right) \cdot \exp \left(-\epsilon_{n} \cdot N\left(r_{n}\right)\right)=\tau_{n} \text { (say) }
$$

where $\epsilon_{n} \rightarrow 0$ as $n \rightarrow \infty$. For $\left|1-\left(z / r_{n}\right)^{m_{n}}\right|$ exceeds $\frac{1}{2} \tau_{n} \cdot m_{n} \cdot\left(r_{n}\right)^{-1}$ outside the circular neighborhoods of the zeros when $n$ is large enough. 
3. By placing more stringent conditions on the moduli of the zeros of $f(z)$, it is possible to relax the symmetrical distribution of their arguments without destroying (13).

Suppose that in addition to the conditions (2), (3) and (4) the sequences $r_{k}$ and $m_{k}$ satisfy

$$
r_{k+1} / r_{k} \geqq\left(1+\frac{1}{k}\right)^{\sigma} \quad \text { for some } \sigma>1,
$$

$$
\lim \sup \log \left(m_{1}+m_{2}+\cdots+m_{n}\right) / \log r_{n}=\rho>\frac{1}{\sigma} .
$$

Let $z_{k, 8}$ be equal to $r_{k} \exp \left(i \theta_{k, s}\right)$, where

$$
2 \pi(s-1) / m_{k} \leqq \theta_{k, \mathrm{e}}<2 \pi s / m_{k}, \quad\left(s=1,2, \cdots, m_{k}\right) .
$$

Let

$$
p_{k}(z)=\prod_{s=1}^{m_{k}}\left(1-\frac{z}{z_{k, s}}\right) .
$$

Let the points $A_{1}, A_{2}, \cdots, A_{n}$ on $|\zeta|=R$ be the corners of a regular polygon of $n$ sides. Let $B_{1}, B_{2}, \cdots, B_{n}$ be points on the $\operatorname{arcs} A_{1} A_{2}$, $A_{2} A_{3}, \cdots, A_{n} A_{1}$ respectively. Let $P$ be any point, with affix $z$, in the $\zeta$-plane. As $|\zeta-z|$ has but one maximum and one minimum on $|\zeta|=R$, we have

$$
\frac{\min \left(P A_{k}\right)}{\max \left(P B_{k}\right)} \leqq \frac{P A_{1} \cdot P A_{2} \cdot P A_{3} \cdots P A_{n}}{P B_{1} \cdot P B_{2} \cdot P B_{3} \cdots P B_{n}} \leqq \frac{\max \left(P A_{k}\right)}{\min \left(P B_{k}\right)} .
$$

Hence, for $r=|z| \neq r_{k}$,

Since

$$
\left|\frac{r-r_{k}}{r+r_{k}}\right| \leqq \frac{\left|p_{k}(z)\right|}{\left|1-\left(z / r_{k}\right)^{m_{k}}\right|} \leqq\left|\frac{r+r_{k}}{r-r_{k}}\right| .
$$

$$
\prod_{k=1}^{\infty} \frac{r-r_{k}}{r+r_{k}}
$$

is convergent, by hypotheses (14), it follows that

$$
F(z)=\prod_{k=1}^{\infty} p_{k}(z)
$$

is convergent and for

$$
\left(r_{n-1} r_{n}\right)^{1 / 2} \leqq r \leqq\left(r_{n} r_{n+1}\right)^{1 / 2},
$$

we obtain the estimate 
$\log |F(z)|-\log |f(z)|=O\left(\left|\log \prod_{k=1}^{n-1}\left[\left(r-r_{k}\right) /\left(r+r_{k}\right)\right]\right|\right)$

$$
\begin{aligned}
& +O\left(\left|\log \prod_{k=n+1}^{\infty}\left[\left(r_{k}-r\right) /\left(r_{k}+r\right)\right]\right|\right) \\
& +\log \left|p_{n}(z)\right|-\log \left|1-\left(z / r_{n}\right)^{m_{n}}\right| .
\end{aligned}
$$

If $m=n-1$, then, by (14)

$$
\begin{aligned}
1 & >\prod_{k=1}^{n-2} \frac{r-r_{k}}{r+r_{k}}>\prod_{k=1}^{m-1} \frac{r_{m}-r_{k}}{r_{m}+r_{k}} \\
& >\prod\left(1-\left(r_{k} / r_{m}\right)^{2}\right)>\prod\left(1-(k / m)^{\sigma}\right)^{2} \\
& >\prod_{k=1}^{m-1}(1-(k / m))^{2}=\left(\frac{m !}{m^{m}}\right)^{2}>e^{-2 m}>e^{-2 n}
\end{aligned}
$$

Also, by (16) and (14) we have

$$
\begin{aligned}
\frac{r-r_{n}}{r+r_{n}} & >\frac{1}{4}\left(1-\left(r_{n-1} / r_{n}\right)\right) \\
& >\frac{1}{4}\left(1-\left(\frac{n-1}{n}\right)^{\sigma}\right) \\
& >\frac{1}{4 n} .
\end{aligned}
$$

Hence

$$
\log \prod_{k=1}^{n-1} \frac{r-r_{k}}{r+r_{k}}=O(n)
$$

Similarly

$$
\log \prod_{k=n+1}^{2 n} \frac{r_{k}-r}{r_{k}+r}=O(n)
$$

Finally

$$
\begin{aligned}
O & >\log \prod_{k>2 n} \frac{r_{k}-r}{r_{k}+r}>2 \sum \log \left(1-\frac{r}{r_{k}}\right) \\
& >-A \cdot \sum_{k>2 n}\left(r / r_{k}\right)>-A \cdot \sum_{k>2 n}\left(r_{n+1} / r_{k}\right) \\
& >-A \sum_{k>2 n}\left(\frac{n+1}{k}\right)^{\sigma}=O(n) .
\end{aligned}
$$


Using these estimates in (17), we see that

$$
\log |F(z)|=\log |f(z)|+\log \left|p_{n}(z)\right|-\log \left|1-\left(\frac{z}{r_{n}}\right)^{m_{n}}\right|+O(n) .
$$

Combined with the estimates (15) and (13) this shows that

$$
\log |F(z)| \sim N(r)
$$

outside small pits around the zeros.

\section{REFERENCE}

1. J. E. Littlewood and A. C. Offord, On the distribution of zeros and a-values of a random integral function. II, Ann. of Math. (2) 49 (1948), 885-952.

G. M. College, Sambalpur, India.

\section{APPROXIMATE FUNCTIONAL APPROXIMATIONS AND THE RIEMANN HYPOTHESIS}

\section{ROBERT SPIRA}

1. Introduction. Using the functional equation for the Riemann zeta function

$$
\zeta(s)=\chi(s) \zeta(1-s)
$$

where

$$
1 / \chi(s)=(2 \pi)^{-s} 2 \cos (\pi s / 2) \Gamma(s),
$$

it was shown in Spira [1] that

(3) $\zeta(s) \neq 0,1 / 2<\sigma<1, \quad t \geqq 10$ implies $|\zeta(1-s)|>|\zeta(s)|$

where $s=\sigma+i$. Using similar but improved techniques, Schoenfeld and Dixon [2] strengthened the result (3) to assuming only $\sigma>1 / 2$, $|t| \geqq 6.8$ and $\zeta(s) \neq 0$. It easily follows from this inequality that the Riemann hypothesis is equivalent to the inequality $|\zeta(1-s)|$ $>|\zeta(s)|, 1 / 2<\sigma<1, t \geqq 10$.

Consider now the formula for $\zeta(s)$ which gives rise to the approximate functional equation and the Riemann-Siegel formula:

Presented to the Society, January 24, 1966 under the title Zeros of approximate functional approximations; received by the editors August 6, 1965 and, in revised form, October 26, 1965. 